\title{
SOME COMPLETELY MONOTONIC FUNCTIONS INVOLVING THE GAMMA AND POLYGAMMA FUNCTIONS
}

\author{
FENG QI ${ }^{\top 9}$, BAI-NI GUO and CHAO-PING CHEN
}

(Received 6 March 2004; revised 21 December 2004)

Communicated by A. J. Pryde

\begin{abstract}
The function $[\Gamma(x+1)]^{1 / x}(1+1 / x)^{x} / x$ is strictly logarithmically completely monotonic in $(0, \infty)$. The function $\psi^{\prime \prime}(x+2)+\left(1+x^{2}\right) / x^{2}(1+x)^{2}$ is strictly completely monotonic in $(0, \infty)$.

2000 Mathematics subject classification: primary 33B15; secondary $26 \mathrm{D} 07$.

Keywords and phrases: completely monotonic function, logarithmically completely monotonic function, gamma function, polygamma function.
\end{abstract}

\section{Introduction}

It is well known that the classical Euler gamma function $\Gamma(z)$ is defined for $\operatorname{Re} z>0$ as

$$
\Gamma(z)=\int_{0}^{\infty} t^{z-1} e^{-t} \mathrm{~d} t
$$

The psi or digamma function $\psi(x)=\Gamma^{\prime}(x) / \Gamma(x)$, the logarithmic derivative of the gamma function, and the polygamma functions can be expressed for $x>0$ and $k \in \mathbb{N}$ as

$$
\psi(x)=-\gamma+\sum_{n=0}^{\infty}\left(\frac{1}{1+n}-\frac{1}{x+n}\right),
$$

The authors were supported in part by the NSF grant 10001016 , the SF grant 0112000200 for the Prominent Youth of Henan Province, the SF of Project for Fostering Innovation Talents at Universities of Henan Province, and the Doctor Fund of Henan Polytechnic University, China.

(c) 2006 Australian Mathematical Society 1446-7887/06 $\$ A 2.00+0.00$ 


$$
\begin{aligned}
\psi^{(k)}(x) & =(-1)^{k+1} k ! \sum_{i=0}^{\infty} \frac{1}{(x+i)^{k+1}}, \\
\psi(x) & =-\gamma+\int_{0}^{\infty} \frac{e^{-t}-e^{-x t}}{1-e^{-t}} \mathrm{~d} t, \\
\psi^{(k)}(x) & =(-1)^{k+1} \int_{0}^{\infty} \frac{t^{k} e^{-x t}}{1-e^{-t}} \mathrm{~d} t,
\end{aligned}
$$

where $\gamma=0.57721566490153286 \ldots$ is the Euler-Mascheroni constant.

DEFINITION 1. A function $f$ is said to be completely monotonic on an interval $l$ if $f$ has derivatives of all orders on $I$ which alternate successively in sign, that is,

$$
(-1)^{n} f^{(n)}(x) \geq 0
$$

for $x \in I$ and $n \geq 0$. If inequality (6) is strict for all $x \in I$ and for all $n \geq 0$, then $f$ is said to be strictly completely monotonic.

DEFINITION 2. A function $f$ is said to be logarithmically completely monotonic on an interval $I$ if its logarithm $\ln f$ satisfies

$$
(-1)^{k}[\ln f(x)]^{(k)} \geq 0
$$

for $k \in \mathbb{N}$ on $I$. If inequality (7) is strict for all $x \in I$ and for all $k \in \mathbb{N}$, then $f$ is said to be strictly logarithmically completely monotonic.

The concepts of (logarithmically) completely monotonic function are defined on an arbitrary interval $I$ here, but the main case is when $I=(0, \infty)$, where the completely monotonic functions are characterized by Bernstein's Theorem [8, page 161] as the Laplace transforms of positive measure $\mu$ in $(0, \infty)$. Bernstein's Theorem states that a function $f$ is completely monotonic in $(0, \infty)$ if and only if

$$
f(x)=\int_{0}^{\infty} e^{-x s} \mathrm{~d} \mu(s),
$$

where $\mu(s)$ is a nonnegative measure, or say that $\mu(s)$ is nondecreasing, on $(0, \infty)$ such that the integral converges for all $x>0$. Hence we conclude that a completely monotonic function which is non-identically zero cannot vanish at any point in $(0, \infty)$. It is clear that a completely monotonic function $f$ in $(0, \infty)$ is strictly completely monotonic if and only if $\mu(s)$ has mass in the open interval $(0, \infty)$. Therefore the sharpenings with 'strict' in Definition 1 and Definition 2 are not very interesting.

To the best of our knowledge, the terminology or the notion 'logarithmically completely monotonic function' was explicitly introduced in $[5,6,7]$ and it was also 
proved in $[5,6]$ that a logarithmically completely monotonic function is completely monotonic. However, it cannot be said to be new, since in [2] this notion appears implicitly in Lemma 2.4 (ii) which can be rephrased as [5, Theorem 1] or [6, Theorem 4].

Completely monotonic functions have applications in many branches. For example, they play a role in potential theory, probability theory, physics, numerical and asymptotic analysis, and combinatorics. Some related references are listed in [1].

It is easy to prove that the function $(1+1 / x)^{-x}$ is completely monotonic in $(0, \infty)$ through proving that it is logarithmically completely monotonic in $(0, \infty)$. A stronger result that the function $(1+1 / x)^{-x}$ is a Stieltjes transform in $(0, \infty)$ follows from [1, Remark 3, page 457]. A function $f$ is called a Stieltjes transform if it is of the form

$$
f(x)=a+\int_{0}^{\infty} \frac{\mathrm{d} \mu(s)}{s+x}
$$

where $a \geq 0$ and $\mu$ is a nonnegative measure on $[0, \infty)$ satisfying

$$
\int_{0}^{\infty} \frac{1}{1+s} \mathrm{~d} \mu(s)<\infty
$$

From (9) we can see directly that a Stieltjes transform is a completely monotonic function.

Among other things, the following results were obtained in [6]: For $\alpha \leq 0$, the function $x^{\alpha} /[\Gamma(x+1)]^{1 / x}$ is strictly logarithmically completely monotonic in $(0, \infty)$. For $\alpha \geq 1$, the function $[\Gamma(x+1)]^{1 / x} / x^{\alpha}$ is strictly logarithmically completely monotonic in $(0, \infty)$. It should be noted that a similar but stronger result is contained in $[2$, Theorem 3.2]. The statement of [2] is that the function

$$
\varphi(x)=\frac{1}{x[\Gamma(1+1 / x)]^{x}}
$$

is a Stieltjes transform and hence completely monotonic. However, it is well known (see, for example, [3, page 127]) that if $\varphi(x)$ is a Stieltjes transform, then so is $1 / \varphi(1 / x)$ and this is exactly the function $[\Gamma(x+1)]^{1 / x} / x$, which is then completely monotonic, since it is a Stieltjes transform.

In [4] the following two inequalities are presented: For $x \in(0,1)$, we have

$$
\frac{x}{[\Gamma(x+1)]^{1 / x}}<\left(1+\frac{1}{x}\right)^{x}<\frac{x+1}{[\Gamma(x+1)]^{1 / x}} .
$$

For $x \geq 1$,

$$
\left(1+\frac{1}{x}\right)^{x} \geq \frac{x+1}{[\Gamma(x+1)]^{1 / x}}
$$


Equality in (10) occurs for $x=1$.

It is easy to obtain, using the standard argument, that

$$
\lim _{x \rightarrow \infty} \frac{[\Gamma(x+1)]^{1 / x}}{x}\left(1+\frac{1}{x}\right)^{x}=1 .
$$

Out of curiosity, the (logarithmically) completely monotonic property of the quotient between two (logarithmically) completely monotonic functions (Stieltjes transforms) $[\Gamma(x+1)]^{1 / x} / x$ and $(1+1 / x)^{-x}$ will be considered in this article. The main result of this consideration is

THEOREM 1.1. The function $x^{-1}(\Gamma(x+1))^{1 / x}(1+1 / x)^{x}$ is strictly logarithmically completely monotonic in $(0, \infty)$.

As a direct consequence of the proof of Theorem 1.1, we have

COROLLARY 1.2. The function

$$
\psi^{\prime \prime}(x)+\frac{x^{4}+5 x^{3}+7 x^{2}+7 x+2}{x^{3}(x+1)^{3}}=\psi^{\prime \prime}(x+2)+\frac{1+x^{2}}{x^{2}(1+x)^{2}}
$$

is strictly completely monotonic in $(0, \infty)$.

\section{Proof of Theorem 1.1}

Define

$$
F(x)=\frac{[\Gamma(x+1)]^{1 / x}}{x^{c}}\left(1+\frac{a}{x}\right)^{x+b}
$$

for $x>0$ and some fixed real numbers $a, b$ and $c$.

Taking the logarithm of $F(x)$ and differentiating yields

$$
\begin{aligned}
\ln F(x)= & (x+b) \ln \left(1+\frac{a}{x}\right)+\frac{\ln \Gamma(x+1)}{x}-c \ln x \\
{[\ln F(x)]^{\prime}=} & \ln \left(1+\frac{a}{x}\right)-\frac{a(x+b)}{x(x+a)}+\frac{x \psi(x+1)-\ln \Gamma(x+1)}{x^{2}}-\frac{c}{x}, \quad \text { and } \\
{[\ln F(x)]^{(n)}=} & (-1)^{n-1}(n-1) !(x+b)\left[\frac{1}{(x+a)^{n}}-\frac{1}{x^{n}}\right] \\
& +(-1)^{n}(n-2) ! n\left[\frac{1}{(x+a)^{n-1}}-\frac{1}{x^{n-1}}\right]+\frac{h_{n}(x)}{x^{n+1}}+(-1)^{n}(n-1) ! \frac{c}{x^{n}} \\
= & (-1)^{n}(n-2) !\left[\frac{(n-1)(b+c)-x}{x^{n}}+\frac{x+n a-(n-1) b}{(x+a)^{n}}\right]+\frac{h_{n}(x)}{x^{n+1}},
\end{aligned}
$$


where $n \geq 2, \psi^{(-1)}(x+1)=\ln \Gamma(x+1), \psi^{(0)}(x+1)=\psi(x+1)$, and

$$
\begin{aligned}
& h_{n}(x)=\sum_{k=0}^{n} \frac{(-1)^{n-k} n ! x^{k} \psi^{(k-1)}(x+1)}{k !}, \\
& h_{n}^{\prime}(x)=x^{n} \psi^{(n)}(x+1) \begin{cases}>0, & \text { if } n \text { is odd; } \\
<0, & \text { if } n \text { is even. }\end{cases}
\end{aligned}
$$

Therefore, we have

$$
\begin{aligned}
& (-1)^{n} x^{n+1}[\ln F(x)]^{(n)}+(-1)^{n+1} h_{n}(x) \\
& \quad=(n-2) !\left\{(n-1)(b+c)-x+\frac{x^{n}[x+n a-(n-1) b]}{(x+a)^{n}}\right\} x
\end{aligned}
$$

and

$$
\begin{aligned}
\frac{\mathrm{d}\left\{(-1)^{n} x^{n+1}[\ln F(x)]^{(n)}\right\}}{\mathrm{d} x} & (-1)^{n} x^{n} \psi^{(n)}(x+1)+(n-2) !\{(n-1)(b+c)-2 x \\
= & \left.\frac{x^{n}\left[a\left(b+a n+a n^{2}-b n^{2}\right)+(2 a+b+2 a n-b n) x+2 x^{2}\right]}{(x+a)^{n+1}}\right\} \\
& +x^{n}\left\{(-1)^{n} \psi^{(n)}(x+1)+(n-2) !\left[\frac{(n-1)(b+c)-2 x}{x^{n}}\right.\right. \\
& \left.\left.+\frac{a\left(b+a n+a n^{2}-b n^{2}\right)+(2 a+b+2 a n-b n) x+2 x^{2}}{(x+a)^{n+1}}\right]\right\} \\
= & x^{n}\left\{(-1)^{n} \psi^{(n)}(x)+\frac{n !}{x^{n+1}}+(n-2) !\left[\frac{(n-1)(b+c)-2 x}{x^{n}}\right.\right. \\
& \left.\left.+\frac{a\left(b+a n+a n^{2}-b n^{2}\right)+(2 a+b+2 a n-b n) x+2 x^{2}}{(x+a)^{n+1}}\right]\right\} .
\end{aligned}
$$

By letting $a=c=1$ and $b=0$, we have

$$
\begin{aligned}
& \frac{\mathrm{d}\left\{(-1)^{n} x^{n+1}[\ln F(x)]^{(n)}\right\}}{\mathrm{d} x} \\
& =x^{n}\left\{(-1)^{n} \psi^{(n)}(x)+\frac{n !}{x^{n+1}}\right. \\
& \left.+(n-2) !\left[\frac{n-1-2 x}{x^{n}}+\frac{n(n+1)+2(n+1) x+2 x^{2}}{(x+1)^{n+1}}\right]\right\} \\
& =x^{n}\left\{(-1)^{n} \psi^{(n)}(x)+(n-2) !\left[\frac{n(n-1)+(n-1) x-2 x^{2}}{x^{n+1}}\right.\right. \\
& \left.\left.\quad+\frac{n(n+1)+2(n+1) x+2 x^{2}}{(x+1)^{n+1}}\right]\right\}
\end{aligned}
$$




$$
\triangleq x^{n}\left\{(-1)^{n} \psi^{(n)}(x)+(n-2) ! g_{n}(x)+(n-2) ! h_{n}(x)\right\} \text {. }
$$

By induction, it follows that $g_{n}^{\prime}(x)=-(n-1) g_{n+1}(x)$ and $h_{n}^{\prime}(x)=-(n-1) h_{n+1}(x)$. This implies $g_{2}^{(n-2)}(x)=(-1)^{n}(n-2) ! g_{n}(x)$ and $h_{2}^{(n-2)}(x)=(-1)^{n}(n-2) ! h_{n}(x)$. Therefore,

$$
\frac{\mathrm{d}\left\{(-1)^{n} x^{n+1}[\ln F(x)]^{(n)}\right\}}{\mathrm{d} x}=(-1)^{n} x^{n}\left[\psi^{\prime \prime}(x)+g_{2}(x)+h_{2}(x)\right]^{(n-2)} .
$$

It is a well-known fact that, for $x>0$ and $r>0$,

$$
\frac{1}{x^{r}}=\frac{1}{\Gamma(r)} \int_{0}^{\infty} t^{r-1} e^{-x t} \mathrm{~d} t
$$

From formulae (3), (5) and (12), for $x \in(0, \infty)$ and any nonnegative integer $i$, we have

$$
\begin{aligned}
\phi(x) \triangleq & \triangleq \\
= & \psi^{\prime \prime}(x)+\frac{x^{4}+5 x^{3}+7 x^{2}+7 x+2}{x^{3}(x+1)^{3}} \\
= & \psi^{\prime \prime}(x)+\frac{2}{x^{3}}+\frac{1}{x^{2}}-\frac{2}{x}+\frac{2}{(1+x)^{3}}+\frac{2}{(1+x)^{2}}+\frac{2}{1+x} \\
= & \frac{1}{x^{2}}-\frac{2}{x}+\frac{2}{(1+x)^{2}}+\frac{2}{1+x}-2 \sum_{i=2}^{\infty} \frac{1}{(x+i)^{3}} \\
= & \psi^{\prime \prime}(x+2)+\frac{1}{x^{2}}-\frac{2}{x}+\frac{2}{(1+x)^{2}}+\frac{2}{1+x}=\psi^{\prime \prime}(x+2)+\frac{1 x^{3}}{x^{2}(1+x)^{2}} \\
= & \int_{0}^{\infty} t e^{-x t} \mathrm{~d} t-2 \int_{0}^{\infty} e^{-x t} \mathrm{~d} t+2 \int_{0}^{\infty} t e^{-(x+1) t} \mathrm{~d} t \\
& +2 \int_{0}^{\infty} e^{-(x+1) t} \mathrm{~d} t-\int_{0}^{\infty} \frac{t^{2} e^{-(x+2) t}}{1-e^{-t}} \mathrm{~d} t \\
= & \int_{0}^{\infty}\left[t-2+(t+4) e^{-t}-\left(t^{2}+2 t+2\right) e^{-2 t}\right] \frac{e^{-x t}}{1-e^{-t}} \mathrm{~d} t \triangleq \int_{0}^{\infty} \frac{q(t) e^{-x t}}{1-e^{-t}} \mathrm{~d} t, \\
\phi^{(i)}(x)= & (-1)^{i} \int_{0}^{\infty} q(t) \frac{t^{i} e^{-x t}}{1-e^{-t}} \mathrm{~d} t,
\end{aligned}
$$

and

$$
\begin{aligned}
q^{\prime}(t) & =\left(2+2 t+2 t^{2}-3 e^{t}+e^{2 t}-t e^{t}\right) e^{-2 t} \triangleq p(t) e^{-2 t}, \\
p^{\prime}(t) & =2+4 t-4 e^{t}+2 e^{2 t}-t e^{t}, \quad p^{\prime \prime}(t)=4-5 e^{t}+4 e^{2 t}-t e^{t} \\
p^{\prime \prime \prime}(t) & =\left(8 e^{t}-t-6\right) e^{t}>0 .
\end{aligned}
$$


Hence, $p^{\prime \prime}(t)$ increases in $(0, \infty)$. Since $p^{\prime \prime}(0)=3>0$, we have $p^{\prime \prime}(t)>0$ and $p^{\prime}(t)$ is increasing. Because $p^{\prime}(0)=0$, it follows that $p^{\prime}(t)>0$ in $(0, \infty)$, and then $p(t)$ is increasing. From $p(0)=0$, it is deduced that $p(t)>0$ and $q^{\prime}(t)>0$ in $(0, \infty)$, then $q(t)$ increases. As a result of $q(0)=0$, we obtain $q(t)>0$ in $(0, \infty)$. Therefore, we have $\phi(x)>0$ in $(0, \infty)$, and then for all nonnegative integer $i$, we have $(-1)^{i} \phi^{(i)}(x)>0$ in $(0, \infty)$. This means that the function $\psi^{\prime \prime}(x)+g_{2}(x)+h_{2}(x)$ is strictly completely monotonic in $(0, \infty)$.

Thus the function $(-1)^{n} x^{n+1}[\ln F(x)]^{(n)}$ is increasing in $x \in(0, \infty)$. Since

$$
\lim _{x \rightarrow 0}\left\{(-1)^{n} x^{n+1}[\ln F(x)]^{(n)}\right\}=0,
$$

we have $(-1)^{n} x^{n+1}[\ln F(x)]^{(n)}>0$, then $(-1)^{n}[\ln F(x)]^{(n)}>0$ for $n \geq 2$ in $(0, \infty)$. Since $[\ln F(x)]^{\prime \prime}>0$, the function $[\ln F(x)]^{\prime}$ is increasing. It is not difficult to obtain $\lim _{x \rightarrow \infty}[\ln F(x)]^{\prime}=0$, so $[\ln F(x)]^{\prime}<0$ and $\ln F(x)$ is decreasing in $(0, \infty)$. In conclusion, the function $\ln F(x)$ is strictly completely monotonic in $(0, \infty)$. The proof is complete.

\section{An open problem}

We would like to pose the following open problem:

OPEN PROBLEM. Under what conditions on $a, b$ and $c$ is the function $F(x)$ defined by (11) completely monotonic, or logarithmically completely monotonic, or a Stieltjes transform on $(0, \infty)$ ?

In some subsequent papers, we will discuss the above open problem and publish its solutions.

\section{Acknowledgements}

The authors would like to express heartily their thanks to he anonymous referee for his/her valuable comments on this paper.

\section{References}

[1] H. Alzer and C. Berg, 'Some classes of completely monotonic functions', Ann. Acad. Sci. Fenn. Math. 27 (2002), 445-460.

[2] —. 'Some classes of completely monotonic functions, II', Ramanujan J., to appear, available online at http://www.math.ku.dkJ $\sim$ berg. 
[3] C. Berg and G. Forst, Potential theory on locally compact Abelian groups, Ergebnisse der Math. 87 (Springer, Berlin, 1975).

[4] Ch.-P. Chen and F. Qi, 'Inequalities relating to the gamma function', Austral. J. Math. Anal. Appl. (1) 1 (2004), Art. 3.

[5] F. Qi and Ch.-P. Chen, 'A complete monotonicity property of the gamma function', J. Math. Anal. Appl. 296 (2004), 603-607.

[6] F. Qi and B.-N. Guo, 'Complete monotonicities of functions involving the gamma and digamma functions', RGMIA Res. Rep. Coll. (1) 7 (2004), Art. 8.

[7] F. Qi, B.-N. Guo and Ch.-P. Chen, 'Some completely monotonic functions involving the gamma and polygamma functions', RGMIA Res. Rep. Coll. (1) 7 (2004), Art. 5.

[8] D. V. Widder, The Laplace transform (Princeton University Press, Princeton, 1941).

Research Institute of Mathematical Inequality Theory

Henan Polytechnic University

Jiaozuo City

Henan Province 454010

China

e-mail: qifeng@hpu.edu.cn

guobaini@hpu.edu.cn

chenchaoping@hpu.edu.cn 